\title{
Rule Out (R/O) Intracranial Aneurysm
}

When imaging patients for intracranial aneurysm, the goals are: (1) to assess the contour of the intracranial arteries, particularly in the regions of the ACOM (anterior communicating artery), PCOM (posterior communicating artery), ICA (internal carotid artery) bifurcation, MCA (middle cerebral artery) trifurcation, basilar tip, and PICA (posterior inferior cerebellar artery); (2) to assess the anatomy of the Circle of Willis and direction of flow, and; (3) to determine if there is evidence of a recent subarachnoid bleed. The 3-D time-of-flight (TOF) magnetic resonance angiography (MRA) is used for assessing contour and anatomy of the major intracranial arteries. The 2-D phase contrast (PC) sequence is used to determine the direction of flow. A fluid-attenuated inversion recovery (FLAIR) sequence is used to search for evidence of a recent subarachnoid bleed. If vasospasm is a clinical concern, diffusion- and perfusion-weighted imaging are added to the imaging assessment. The following Basic Protocol can be used for the evaluation of stable patients, and requires $20 \mathrm{~min}, 18 \mathrm{sec}$ to complete.

\section{STANDARD IMAGING FOR ANEURYSM DETECTION}

In order to perform the optional sequences for perfusion and diffusion studies, a scanner with echo-planar capabilities is required (Table A1.2.1). MR angiography and the standard anatomical MR imaging sequences included in the protocol do not require these faster gradients. The parameters given here are optimized for a 1.5T General Electric (GE) LX system with software version 8.35 and may require modification if using different software versions, field strengths, or manufacturers.

Data processing is required for the 3-D TOF MRA sequence described in this protocol. For optimal assessment of the volumetric 3-D TOF MRA data, separate volumes of interest (VOI) for the left anterior, right anterior, and posterior circulation should be created. Images from $15^{\circ}$-rotations about the vertical axis should be saved. An additional anterior circulation volume of interest should include both anterior cerebral arteries and the region of the anterior communicating artery for visual inspection. Images from $15^{\circ}$-rotations about the horizontal axis should also be saved. Although maximum intensity projections (MIP) are superior to volume and surface reconstructions for assessment of vascular patency, surface reconstructions can be helpful in visualizing aneurysms. All of our MRA data are processed on a GE Advantage Windows Workstation version 3.1P using standard GE Advantage Windows software or FuncTool version 1.9M. In the hands of an experienced technologist, the postprocessing will require $\sim 5 \mathrm{~min}$.

NOTE: Be sure that technologists and nurses have immediate access to any emergency equipment that may be relevant to a given study, or that may be needed for a particular patient, such as crash carts or oxygen.

Table A1.2.1 Equipment Requirements for Cerebral Vascular Assessment

\begin{tabular}{ll}
\hline Coil type & Quadrature head or neurovascular coil \\
Gradient coil strength & $22 \mathrm{mT} / \mathrm{m}$ (or whatever the system permits) \\
Slew rate & $\geq 120 \mathrm{~T} / \mathrm{m} / \mathrm{sec}$ \\
Cardiac gating & Not necessary \\
Peripheral gating & Not necessary \\
Respirator & If required by patient \\
Oxygen & If required by patient \\
Motion cushions & Useful \\
Use of contrast agents & Yes
\end{tabular}

Contributed by P. Ellen Grant, R. Gilberto Gonzalez, and Pamela W. Schaefer

Current Protocols in Magnetic Resonance Imaging (2002) A1.2.1-A1.2.12

Copyright @ 2002 by John Wiley \& Sons, Inc.
BASIC

PROTOCOL

Intracranial Arterial Disease

A1.2.1

Supplement 6 


\section{Set up patient and equipment}

1. Interview (screen) the patient to ensure that he or she has no counterindications such as cardiac pacemakers or other implants containing ferromagnetic materials. Also be sure to find out if the patient has any health conditions that may require the presence of special emergency equipment during the scanning procedure, or necessitate any other precautions. Do not forget to ask if the patient has any drug allergies and document them.

Generally, standard screening forms are used for all patients scanned in a magnetic resonance system (see APPENDIX 1).

The presence of any ferromagnetic metals may be a health hazard to the patient when he or she is inside the magnet, and will also affect the imaging. If in doubt as to the exact composition of the items, it is best to exclude patients with any metal implants; see Shellock (1996) for discussion of what implants may be safely scanned using magnetic resonance.

Patients may be accompanied into the magnet room by a friend or family member, who can sit in the room during the scan and comfort the patient as needed. This companion must be screened as well to ensure the absence of loose metal objects on the body or clothing.

2. If the procedure is a research protocol, have the patient sign any necessary consent forms.

3. Have the patient remove all jewelry and change into a gown to eliminate any metal that might be found in clothing.

4. Have the patient wash off any mascara and other makeup to avoid local tissue heating and image artifacts.

5. Inform the patient about what will occur during the procedure, what he or she will experience while in the magnet, and how to behave, including the following:

a. If earphones or headphones are used to protect the ears from the loud sounds produced by the gradients, the patient will be asked to wear these, but will be able to communicate with you at any time during the imaging.

b. The patient will be given a safety squeeze-bulb or similar equipment to request assistance at any time (demonstrate how this works).

c. For good results the patient should not talk, and should avoid or minimize swallowing or other movement, during each scan-i.e., as long as the banging sounds continue. Between scans, talking and swallowing are allowed in most cases, but should be avoided when comparative positional studies are being performed; the patient will be informed when this is the case.

d. Nevertheless, the patient may call out at any time if he or she feels that it is necessary.

6. Have the patient mount onto the table. Either before or right after the patient lies down, set up any triggering devices or other monitoring equipment that is to be used.

7. Center the patient in a head or neurovascular coil at the region where the key information is desired. Make sure that the head and neck are constrained to prevent motion, especially if high-resolution scans are to be run.

Generally, the patient's head is fixed so that the head is horizontal (not tilted) and the neck and head lie along the axis of the patient table; other positions may be appropriate depending on the needs at hand.

Most scanners have a special neurovascular coil for MRA; otherwise, a head coil should be used and the patient is placed as far in as possible so that the bifurcation of the main carotid artery into the internal and external carotid arteries can be imaged. 
Table A1.2.2 Rapid Sagittal $T_{1}$-Weighted Scout

\begin{tabular}{ll}
\hline Patient position & Supine \\
Scan type & 2-D gradient echo \\
Imaging plane (orientation) & Sagittal \\
Central slice or volume center & Nasion \\
Echo time $\left(T_{\mathrm{E}}\right)$ & Minimum (at least $4.1 \mathrm{msec})$ \\
Receiver bandwidth $(\mathrm{RBW})$ & $\pm 15.6 \mathrm{kHz}$ \\
Repeat time $\left(T_{\mathrm{R}}\right)$ & $100 \mathrm{msec}$ \\
Flip angle $(\mathrm{FA})$ & $60^{\circ}$ \\
Fields of view $\left(\mathrm{FOV}_{\mathrm{x}}, \mathrm{FOV}_{\mathrm{y}}\right)$ & $240 \mathrm{~mm}, 240 \mathrm{~mm}$ \\
Resolution $(\Delta x, \Delta y)$ & $0.94 \mathrm{~mm}, 1.88 \mathrm{~mm}$ \\
Number of data points collected $\left(N_{\mathrm{x}}, N_{\mathrm{y}}\right)$ & 256,128 \\
Display matrix $\left(D_{\mathrm{x}}, D_{\mathrm{y}}\right)$ & 256,256 \\
Slice thickness $(\Delta z)$ & $7 \mathrm{~mm}$ \\
Number of slices & 7 \\
Slice gap & $2 \mathrm{~mm}$ \\
Number of excitations $(\mathrm{NEX})$ & 1 \\
Number of acquisitions $\left(N_{\mathrm{acq}}\right)$ & 1 \\
Read direction & Anterior-posterior \\
Scan time & $16 \mathrm{sec}$ \\
\hline
\end{tabular}

Table A1.2.3 Transverse FLAIR

\begin{tabular}{|c|c|}
\hline Patient position & Supine \\
\hline Scan type & Inversion recovery, fast-spin echo \\
\hline Imaging plane (orientation) & Transverse \\
\hline Variable bandwidth & Yes \\
\hline Central slice or volume center & Nasion \\
\hline Echo time $\left(T_{\mathrm{E}}\right)$ & $140 \mathrm{msec}$ \\
\hline Receiver bandwidth (RBW) & $\pm 20.83 \mathrm{kHz}$ \\
\hline Echo train length (ETL) & 12 (not selectable on GE's system) \\
\hline Repeat time $\left(T_{\mathrm{R}}\right)$ & $10,000 \mathrm{msec}$ \\
\hline Inversion time $\left(T_{\mathrm{I}}\right)$ & $2200 \mathrm{msec}$ \\
\hline Flip angle (FA) & $180^{\circ}$ \\
\hline Fields of view $\left(\mathrm{FOV}_{\mathrm{x}}, \mathrm{FOV}_{\mathrm{y}}\right)$ & $220 \mathrm{~mm}, 165 \mathrm{~mm}$ \\
\hline Resolution $(\Delta x, \Delta y)$ & $0.86 \mathrm{~mm}, 0.86 \mathrm{~mm}$ \\
\hline Number of data points collected $\left(N_{\mathrm{x}}, N_{\mathrm{y}}\right)$ & 256,192 \\
\hline Display matrix $\left(D_{\mathrm{x}}, D_{\mathrm{y}}\right)$ & 256,256 \\
\hline Slice thickness $(\Delta z)$ & $5 \mathrm{~mm}$ \\
\hline Number of slices & 12 \\
\hline Slice gap & 1 \\
\hline Number of excitations (NEX) & 1 \\
\hline Number of acquisitions $\left(N_{\text {acq }}\right)$ & 2 \\
\hline Read direction & Anterior-posterior \\
\hline Scan time & $5 \mathrm{~min}, 20 \mathrm{sec}$ \\
\hline
\end{tabular}


Table A1.2.4 Transverse 3-D TOF MRA

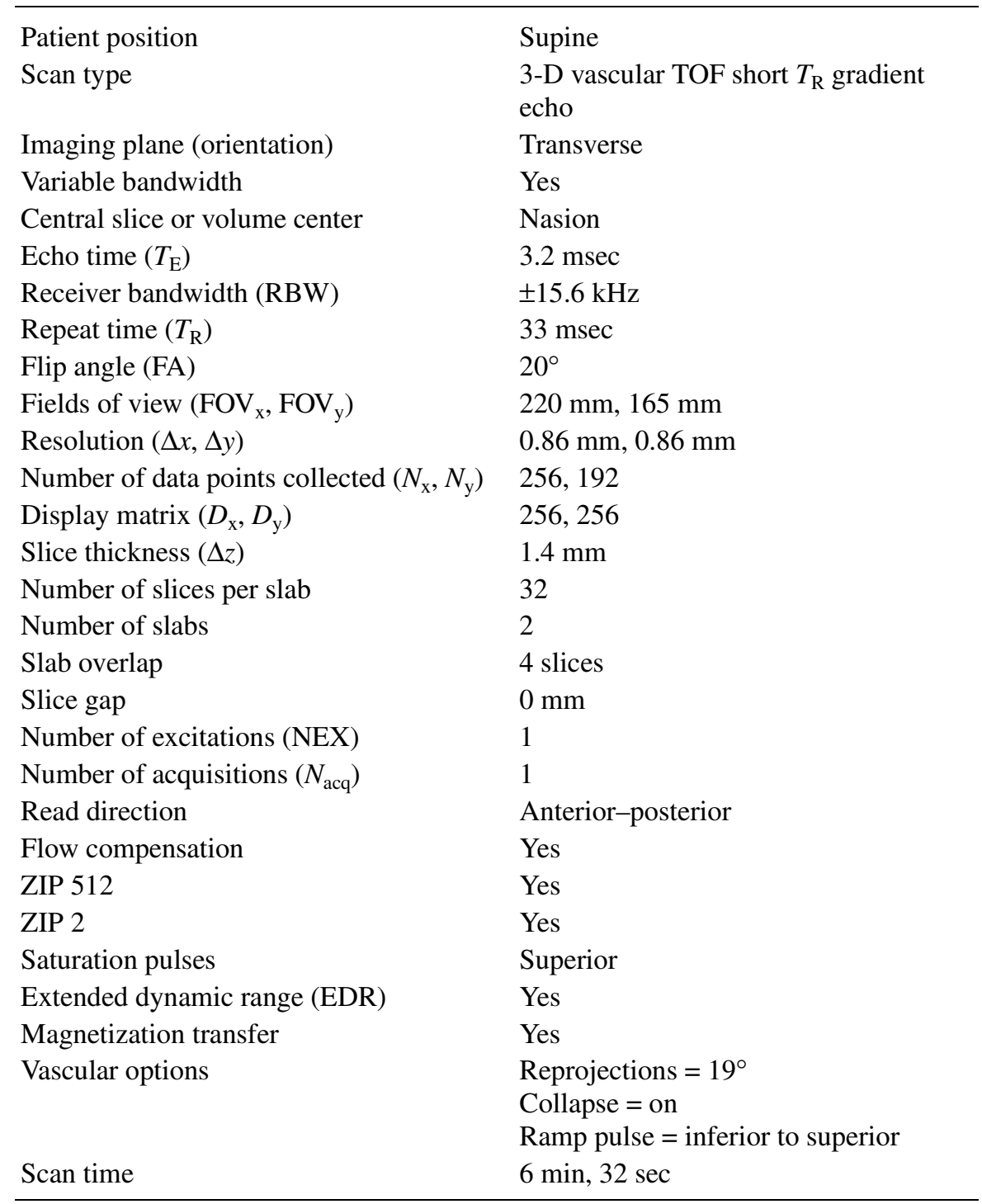

8. If needed, place a pillow or other support under the knees to make the patient more comfortable.

9. Place the patient such that the laser light is centered over the patient's nasion, and put him or her into the center of the magnet.

Once this step has been performed, so long as the patient does not move on the table, the table itself can be moved and then replaced in the same position as before without jeopardizing the positioning of one scan relative to another.

10. If the patient is unable to hold still, provide an appropriate sedative.

\section{Sequence 1: Rapid sagittal $T_{1}$-weighted scout}

11. To validate the patient's position, perform a fast sagittal scout scan using the imaging parameters in Table A1.2.2. (Some medical centers may prefer a rapid three-plane scout instead of this multi-slice sagittal scout.) 


\begin{tabular}{ll}
\hline Patient position & Supine \\
Scan type & 2-D vascular phase contrast gradient echo \\
Imaging plane (orientation) & Transverse \\
Central slice or volume center & Nasion \\
Echo time $\left(T_{\mathrm{E}}\right)$ & $6 \mathrm{msec}$ \\
Repeat time $\left(T_{\mathrm{R}}\right)$ & $20 \mathrm{msec}$ \\
Flip angle $(\mathrm{FA})$ & $20^{\circ}$ \\
Fields of view $\left(\mathrm{FOV}, \mathrm{FOV}_{\mathrm{y}}\right)$ & $220 \mathrm{~mm}, 165 \mathrm{~mm}$ \\
Resolution $(\Delta x, \Delta y)$ & $0.86 \mathrm{~mm}, 1.29 \mathrm{~mm}$ \\
Number of data points collected $\left(N_{\mathrm{x}}, N_{\mathrm{y}}\right)$ & 256,128 \\
Display matrix $\left(D_{\mathrm{x}}, D_{\mathrm{y}}\right)$ & 256,256 \\
Slice thickness $(\Delta z)$ & $10 \mathrm{~mm}$ \\
Number of slices & 3 \\
Slice gap & 0 mm \\
Number of excitations $(\mathrm{NEX})$ & 4 \\
Number of acquisitions $\left(N_{\mathrm{acq}}\right)$ & 3 \\
Read direction & Anterior-posterior \\
Flow compensation & Yes \\
Slice series & sequential \\
Vascular options & Flow reconstruction type = phase difference \\
Scan time & Velocity encoding $=80 \mathrm{~cm} / \mathrm{sec}$ \\
& Acquired flow directions $=$ all \\
& Collapse $=$ on \\
& Flow analysis $=$ off \\
& \\
&
\end{tabular}

\section{Sequence 2: Transverse FLAIR}

12. From the sagittal scout, select the image through the center of the brain to set up the locations for the transverse FLAIR sequence. The locations should be the same as those chosen for the transverse diffusion sequence (if it will be run at a latter time). Set up the imaging parameters according to Table A1.2.3.

13. Warn the patient that this sequence is starting and begin the scan.

\section{Sequence 3: Transverse 3-D TOF MRA}

14. From the sagittal scout, select the image through the center of the brain to set up the locations for the transverse 3-D TOF MRA. Set up the imaging parameters according to Table A1.2.4.

Two slabs are posted on the midline sagittal image, centered at the top of the sella tursica.

15. Warn the patient that this sequence is starting and begin the scan.

\section{Data processing and viewing for sequence 3}

16. Once the transverse partitions from the 3-D TOF MRA have been obtained, select three volumes of interest (VOI) that select out the posterior circulation, the right anterior circulation and the left anterior circulation, and rotate the maximum intensity projections or surface reconstructions of these three VOIs through $360^{\circ}$ about the vertical axis, saving images at $15^{\circ}$-intervals. Rotate a fourth VOI, that selects out both anterior circulations, through $360^{\circ}$ about the horizontal axis, saving images at $15^{\circ}$-intervals. 
Table A1.2.6 Transverse $T_{2}$-Weighted FSE

\begin{tabular}{|c|c|}
\hline Patient position & Supine \\
\hline Scan type & Fast-spin echo \\
\hline Imaging plane (orientation) & Transverse \\
\hline Variable bandwidth & Yes \\
\hline Pulse sequence database (PSD) & FSE-XL \\
\hline Central slice or volume center & Nasion \\
\hline Echo time $\left(T_{\mathrm{E}}\right)$ & $102 \mathrm{msec}$ \\
\hline Receiver bandwidth (RBW) & $\pm 11.36 \mathrm{kHz}$ \\
\hline Echo train length (ETL) & 12 \\
\hline Repeat time $\left(T_{\mathrm{R}}\right)$ & $6000 \mathrm{msec}$ \\
\hline Flip angle (FA) & $90^{\circ}$ \\
\hline Fields of view $\left(\mathrm{FOV}_{\mathrm{x}}, \mathrm{FOV}_{\mathrm{y}}\right)$ & $220 \mathrm{~mm}, 165 \mathrm{~mm}$ \\
\hline Resolution $(\Delta x, \Delta y)$ & $0.86 \mathrm{~mm}, 0.86 \mathrm{~mm}$ \\
\hline Number of data points collected $\left(N_{\mathrm{x}}, N_{\mathrm{y}}\right)$ & 256,192 \\
\hline Display matrix $\left(D_{\mathrm{x}}, D_{\mathrm{y}}\right)$ & 256,256 \\
\hline Slice thickness $(\Delta z)$ & $5 \mathrm{~mm}$ \\
\hline Number of slices & 28 \\
\hline Slice gap & $1 \mathrm{~mm}$ \\
\hline Number of excitations (NEX) & 2 \\
\hline Number of acquisitions $\left(N_{\mathrm{acq}}\right)$ & 1 \\
\hline Read direction & Anterior-posterior \\
\hline Flow compensation & Yes \\
\hline Extended dynamic range (EDR) & Yes \\
\hline Scan time & $2 \mathrm{~min}, 48 \mathrm{sec}$ \\
\hline
\end{tabular}

\section{Sequence 4 (optional): Transverse 2-D phase contrast}

Flow direction information may be helpful in determining which vessels contribute flow to the aneurysm.

17. From the sagittal scout, select the image through the center of the brain to set up the locations for the transverse 2-D PC MRA. Set up the imaging parameters according to Table A1.2.5.

Three slices are posted on the midline sagittal image, centered at the top of the sella tursica.

18. Warn the patient that this sequence is starting and begin the scan.

\section{Sequence 5: Transverse $T_{2}$-weighted fast-spin echo (FSE)}

19. From the sagittal scout, select the image through the center of the brain to set up the locations for the transverse $T_{2}$-weighted FSE sequence. The locations should be the same as those chosen for the transverse diffusion sequence (if it will be used). Set up the imaging parameters according to Table A1.2.6.

20. Warn the patient that this sequence is starting and begin the scan. 


\section{ANEURYSM SCREEN AND ASSESSMENT FOR VASOSPASM/INFARCTION}

When there is concern for vasospasm and infarction, the imaging sequences in the Basic Protocol should be performed with the addition of the diffusion and perfusion sequences described below.

\section{Materials}

Normal saline $(0.9 \% \mathrm{NaCl})$, sterile

Gadolinim-DTPA contrast agent (e.g., Magnevist, Omniscan, or Prohance)

\section{Set up equipment and patient}

1. Repeat Basic Protocol, steps 1 to 5.

2. Establish an intravenous (i.v.) line from which the contrast agent can be injected, and attach this line securely to the patient so that movement into or out of the magnet will not pull at the patient's arm.

It is preferable to insert the line prior to imaging and to leave the patient in the magnet, with no intervening motion, between the scans run before contrast agent injection and those run after injection.

3. Connect the MR compatible injection pump that is cleared of air and loaded with a double dose of contrast agent and with saline flush, to the patient. Do a small test injection to ensure that the i.v. is working properly.

4. Program the $5 \mathrm{ml} / \mathrm{sec}$ injection rate for both contrast agent and saline, contrast agent dose required (double dose by weight), saline flush required (typically $40 \mathrm{ml}$ ) and 10 -sec time delay. Start the saline running to keep the vein open, and arm the injection pump. Do not inject the contrast agent at this time.

5. Repeat Basic Protocol, steps 6 to 10.

\section{Sequences 1 to 5}

6. Perform sequences 1 to 5 as described in the Basic Protocol.

\section{Sequence 6: Transverse diffusion}

7. From the sagittal scout, select the image through the center of the brain to set up the locations for the transverse diffusion-weighted sequence. The transverse images should begin at the foramen magnum and end at the top of the brain. Set up imaging parameters according to Table A1.2.7.

8. Warn the patient that this sequence results in loud beeping noises and begin the scan.

\section{Data processing and viewing for sequence 6}

9. Process the diffusion-weighted images.

Most diffusion-weighted sequences will perform a minimum of four sequences: three with orthogonal diffusion gradient directions and one with a minimal or no diffusion gradient. Often, a fifth set of images is also provided that combines the three orthogonal gradient images to produce a set of images whose signal intensity is not affected by the diffusion direction. This is the diffusion-weighted trace image (DWI). In order to obtain Apparent Diffusion Coefficient (ADC) maps the images may need further processing. On a GE system, the ADC maps can be obtained by processing on the Advantage Window Workstation version 3.1P using FuncTool version 1.9M. 
Table A1.2.7 Transverse Diffusion

\begin{tabular}{ll}
\hline Patient position & $\begin{array}{l}\text { Supine } \\
\text { Single-shot spin-echo-EPI (echo } \\
\text { planar imaging), diffusion }\end{array}$ \\
Imaging plane (orientation) & Transverse \\
epi2NV & Nasion \\
Central slice or volume center & $99.3 \mathrm{msec}$ \\
Echo time $\left(T_{\mathrm{E}}\right)$ & $6000 \mathrm{msec}$ \\
Repeat time $\left(T_{\mathrm{R}}\right)$ & $90^{\circ}$ \\
Flip angle $(\mathrm{FA})$ & $220 \mathrm{~mm}, 220 \mathrm{~mm}$ \\
Fields of view $\left(\mathrm{FOV}_{\mathrm{x}}, \mathrm{FOV}_{\mathrm{y}}\right)$ & $1.72 \mathrm{~mm}, 1.72 \mathrm{~mm}$ \\
Resolution $(\Delta x, \Delta y)$ & 128,128 \\
Number of data points collected $\left(N_{\mathrm{x}}, N_{\mathrm{y}}\right)$ & 128,128 \\
Display matrix $\left(D_{\mathrm{x}}, D_{\mathrm{y}}\right)$ & $5 \mathrm{~mm}$ \\
Slice thickness $(\Delta z)$ & 23 \\
Number of slices & $1 \mathrm{~mm}$ \\
Slice gap & 3 \\
Number of excitations $(\mathrm{NEX})$ & 1 \\
Number of acquisitions $\left(N_{\mathrm{acq}}\right)$ & Right-left \\
Read direction & Fat saturation $($ automatic with EPI) \\
Saturation pulses & Ramp sampling = 1, burst sampling =0 \\
Control variables $(\mathrm{CV})$ & $1000 \mathrm{sec} / \mathrm{min}^{2}$ \\
$b$-value & $2 \mathrm{~min}, 38 \mathrm{sec}$ \\
Scan time &
\end{tabular}

Table A1.2.8 Transverse Perfusion

Patient position

Scan type

Pulse sequence database (PSD)

Imaging plane (orientation)

Variable bandwidth

Central slice or volume center

Echo time $\left(T_{\mathrm{E}}\right)$

Receiver bandwidth (RBW)

Repeat time $\left(T_{\mathrm{R}}\right)$

Flip angle (FA)

Fields of view $\left(\mathrm{FOV}_{\mathrm{x}}, \mathrm{FOV}_{\mathrm{y}}\right)$

Resolution $(\Delta x, \Delta y)$

Number of data points collected $\left(N_{\mathrm{x}}, N_{\mathrm{y}}\right)$

Display matrix $\left(D_{\mathrm{x}}, D_{\mathrm{y}}\right)$

Slice thickness $(\Delta z)$

Number of slices

Slice gap

Number of excitations (NEX)

Number of acquisitions $\left(N_{\text {acq }}\right)$

Read direction

Slice location

Multi-phase

Slice series

Control variables $(\mathrm{CV})$

Scan time
Supine

Single-shot spin-echo-EPI

epi2perf

Transverse

Yes

Nasion

$65 \mathrm{msec}$

$\sim 200 \mathrm{kHz}$ (not selectable on GE's system)

$1500 \mathrm{msec}$

$90^{\circ}$

$220 \mathrm{~mm}, 220 \mathrm{~mm}$

$1.72 \mathrm{~mm}, 1.72 \mathrm{~mm}$

128,128

128,128

5 or $6 \mathrm{~mm}$

11

$1 \mathrm{~mm}$

1

1

Left-right

Inferior slice to include MCA

Yes, 46 phases per location, minimum delay between acquisitions

Interleaved

Ramp sampling $=$ on, burst sampling $=$ off

$1 \mathrm{~min}, 11 \mathrm{sec}$ 
10. Using the transverse FSE $T_{2}$-weighted images, find the slice location where the middle cerebral arteries are best seen. Using the imaging parameters as shown in Table A1.2.8, set up the transverse images starting at the same location as the MCA were identified on the $T_{2}$-weighted FSE images. Typically, only 10 or 11 slices can be obtained in the given $T_{\mathrm{R}}$ interval. The slice thickness and gap can be modified so that the perfusion study covers any diffusion abnormality.

11. Check that the injection pump is ready to inject the contrast agent and that the injection is set to start $10 \mathrm{sec}$ after the scan begins.

12. Warn the patient that this sequence is starting and that part of the way through the scan the iv injection will occur. Warn the patient that a cool sensation may be felt during the injection. Begin the scan.

A dose of $0.2 \mathrm{mmol} / \mathrm{kg}$ (which is equivalent to two usual doses) of contrast agent is usually given.

\section{Data processing and viewing for sequence 7}

13. Process the perfusion images on an Advantage Windows Workstation version 3.1P using FuncTool version 1.9M to give maps of relative cerebral blood volume (rCBV), relative cerebral blood flow (rCBF) and mean transit time (MTT).

\section{COMMENTARY}

\section{Background Information}

Subarachnoid hemorrhage (SAH) due to rupture of intracranial aneurysms is a major cause of cerebrovascular disease related death (Whisnant et al., 1982; Ingall et al., 1989). Between $1 \%$ and $7.9 \%$ of the population have unruptured aneurysms (Locksley, 1969; Rosenorn et al., 1988; Atkinson et al., 1989; Ingall et al., 1989; Inagawa and Hirano, 1990; Iwata et al., 1991; Nakagawa and Hashi, 1994; Rinkel et al., 1998) with annual risks of rupture estimated to be $0.5 \%$ (International Study of Unruptured Intracranial Aneurysms Investigators, 1998) to 2.3\% (Yasui et al., 1997). Mortality rates with SAH have been reported to be as high as $52 \%$ to $67 \%$ (Winn et al., 1977; Whisnant et al., 1982; Juvela et al., 1993; Yasui et al., 1997; International Study of Unruptured Intracranial Aneurysms Investigators, 1998). With the advent of MR (and computed tomography; CT) angiography, there has been increasing interest in screening for the presence of aneurysms, particularly in patients with higher risks such as patients with polycystic kidney disease, connective tissue disorders, or two first-degree relatives with intracranial aneurysms. The sensitivity of 3-D TOF MRA at $1.5 \mathrm{~T}$ for detecting aneurysms has been reported to be $75 \%$ dropping to $58 \%$ for aneurysms $<5 \mathrm{~mm}$ (Korogi et al., 1997). When all image sequences are closely scrutinized including the 3-D TOF MRA partition data and MIP, sensitivity has been reported to be $81 \%$ with $100 \%$ specificity in the setting of acute SAH (Wilcock et al., 1996).

In most medical centers, MR (or CT) angiography has replaced catheter angiography in screening patients at risk for intracranial aneurysms and to follow known aneurysms. In patients with acute subarachnoid hemorrhage, it can also be used prior to catheter angiography to plan the optimal projections on catheter angiography and to help visualize the surgical approach. CT angiography is beginning to provide strong competition in this area, especially for presurgical planning where bony landmarks are often helpful to the surgeon.

With the location of aneurysms being most common in the ACOM, MCA bifurcation, PCOM, carotid siphon, basilar tip, and PICA origin, these regions are the main target areas for MR angiography. MR angiography is not sensitive at detecting aneurysms in distal branches and therefore if distal aneurysms, such as mycotic aneurysms, are suspected, catheter angiogram is still the study of choice.

FLAIR imaging is highly sensitive in the detection of subarachnoid hemorrhage in the acute stage (Fig. A1.2.1; Singer et al., 1998; Bakshi et al., 1999) but artifacts from flowing CSF (cerebrospinal fluid) that is not suppressed may be difficult to differentiate from true subarachnoid blood since both are hyperintense. Some authors suggest using proton den-
Intracranial Arterial Disease

A1.2.9

Supplement 6 
A

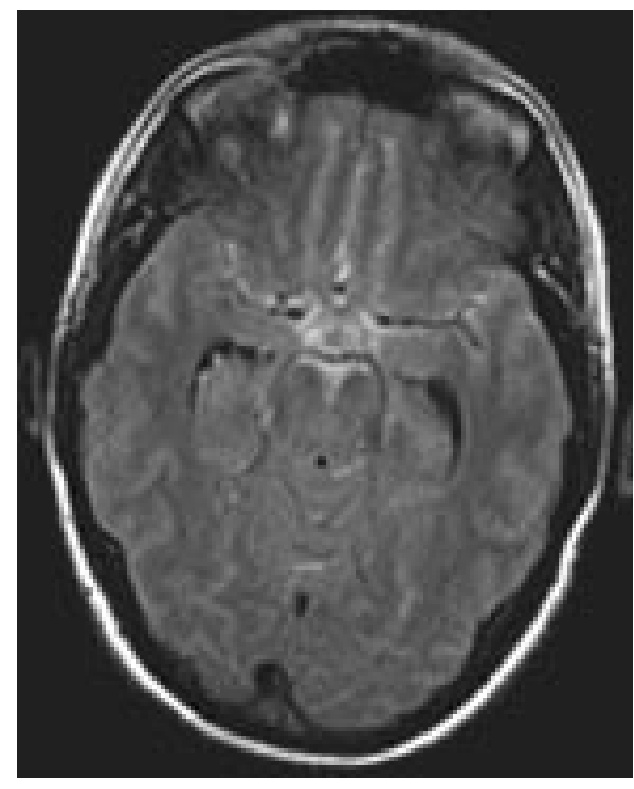

B

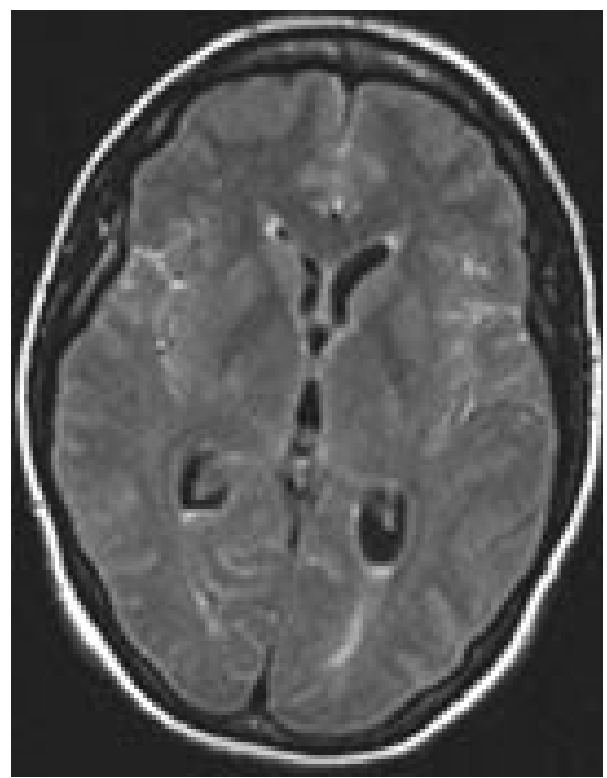

Figure A1.2.1 Transverse FLAIR images at the level of the basal cisterns $(\mathbf{A})$ and level of the basal ganglia (B). Increased signal is seen throughout the subarachnoid spaces including the anterior interhemispheric fissure, basal cisterns and bilateral sylvian fissures. High signal material is also seen layering in the posteriorly in the trigones of lateral ventricles in (B). The high signal is due to acute subarachnoid and intraventricular blood.

Rule Out (R/O) Intracranial Aneurysm

A1.2.10 sity-weighted images in conjunction with FLAIR images to help differentiate flow artifacts from blood since all cases of subarachnoid blood seen on CT were also seen on the proton density-weighted sequence (Wiesmann et al., 1999). The authors find correlation with the $T_{2}$-weighted FSE sequence helpful in distinguishing flow artifacts but still obtain noncontrast CT studies prior to MRI to rule out acute SAH.

In patients with vasospasm, those that were symptomatic showed areas of increased signal on diffusion-weighted imaging consistent with areas of acute ischemia. In these same patients, perfusion-weighted imaging showed larger areas of increased transit time in a distribution that correlated with clinical symptoms (Rordorf, 1999). This study indicated that there was a role for diffusion- and perfusion-weighted imaging in the diagnosis and evaluation of patients with symptomatic vasospasm.

\section{Critical Parameters and Troubleshooting}

Important basic parameters in MR angiography are discussed in UNIT A1.1. If the MIP representations are difficult to interpret, surface reconstructions may improve aneurysm visualization. Also, multiplanar (including curved) reformations may help in optimizing aneurysm visualization, particularly visualization of the aneurysm neck. If there is a large and/or partially thrombosed aneurysm, a post contrast 3-D TOF MRA may be helpful. With the postcontrast MRA, the $T_{\mathrm{R}}$ can be decreased and the flip angle increased compared to the pre-contrast study to improve visualization.

With FLAIR sequences, it is important to make sure that the $T_{\mathrm{R}}$ and $T_{1}$ values have not been modified and that vendor-specific recommendations for optimal CSF suppression have been followed. For example, in GE FLAIR sequences, at least 2 interleaved acquisitions are required for optimal CSF suppression.

\section{Anticipated Results}

The 3-D TOF MRA data should allow accurate $3-\mathrm{D}$ visualization of aneurysms $>3$ $\mathrm{mm}$ in size as well as visualization of the aneurysm neck and relationship to other intracranial structures (Fig. A1.2.2). When MIPs are confusing, multiplanar reforma- 
A

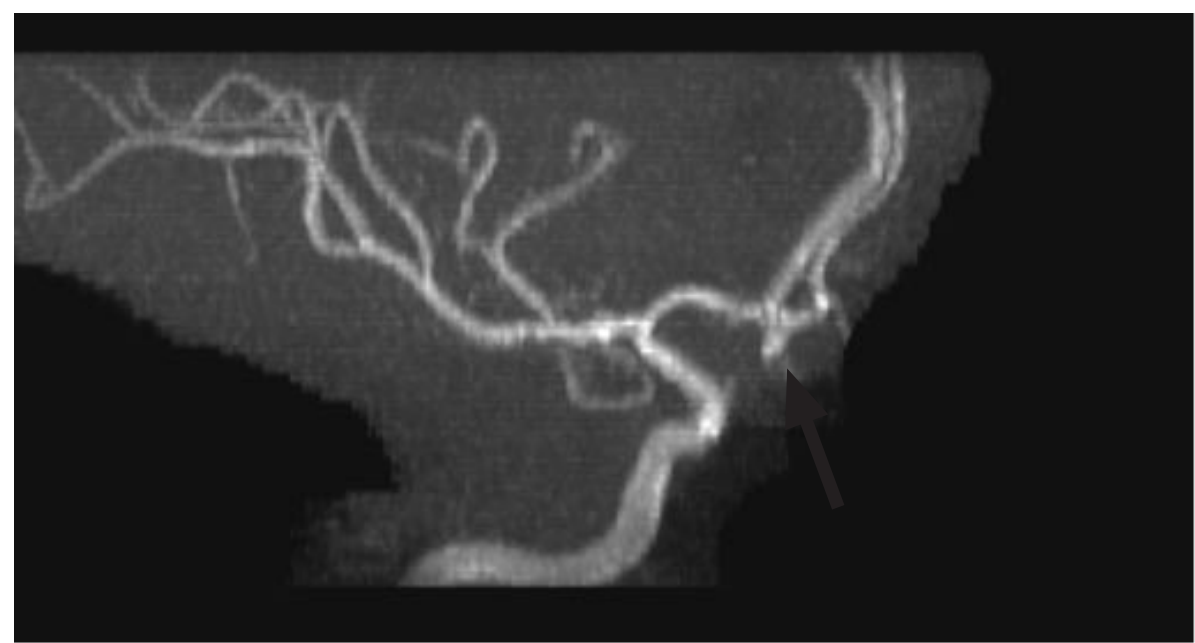

$\mathrm{B}$

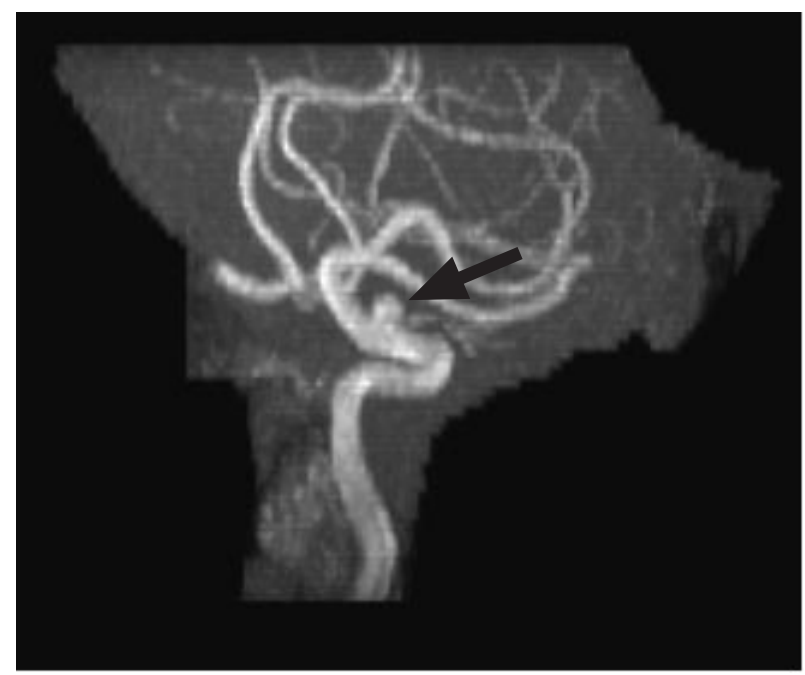

Figure A1.2.2 MIP of the right anterior circulation, ACOM and left A2 segment (A) from the same patient shown in Figure A1.2.1. A small aneurysm arising from the right ACOM/A2 junction, is seen pointing inferiorly and to the right (arrow). (B) MIP of the left anterior circulation in a different patient shows a small aneurysm (arrow) arising from the left ICA just above the origin of the ophthalmic artery.

tions may be helpful to differentiate an infundibulum from an aneurysm and may help in the assessment of the aneurysm neck. Partially thrombosed aneurysms may have regions of intrinsically bright $T_{1}$-weighted signal due to methemaglobin but these can be distinguished from flow related enhancement by viewing the partition data.

\section{Literature Cited}

Atkinson, J.L.D., Sindt, T.M., Houser, O.W., and Whisnant, J.P. 1989. Angiographic frequency of anterior circulation intracranial aneurysms. $J$. Neurosurg. 70:551-555.
Bakshi, R., Kamran, S., Kinkel, P.R., Bates, V.E., Mechtler, L.L., Janardhan, V., Belani, S.L., and Kinkel, W.R. 1999. Fluid-attenuated inversionrecovery MR imaging in acute and subacute cerebral intraventricular hemorrhage. Am. J. Neuroradiol. 20:629-636.

Inagawa, T. and Hirano, A. 1990. Autopsy study of unruptured incidental intracranial aneurysms. Surg. Neurol. 34:361-365.

Ingall, T.J., Whisnant, J.P., Wiebers, D.O., and O'Fallon, W.M. 1989. Has there been a decline in subarachnoid hemorrhage mortality? Stroke 20:718-724.

International Study of Unruptured Intracranial Aneurysms Investigators. 1998. Unruptured in-
Intracranial Arterial Disease 
tracranial aneurysms: Risk of rupture and risks of surgical intervention. N. Engl. J. Med. 339:1725-1733.

Iwata, K., Misu, N., Terada, K., Kawai, S., Momose, M., and Nakagawa, H. 1991. Screening for unruptured asymptomatic intracranial aneurysms in patients undergoing coronary angiography. $J$. Neurosurg. 75:52-55.

Juvela, S., Porras, M., and Heiskanen, O. 1993. Natural history of unruptured intra-cranial aneurysms: A long-term follow-up study. J. Neurosurg. 79:174-182.

Korogi, Y., Takahashi, M., Mabuchi, N., Watabe, T., Shiokawa, Y., Shiga, H., O’Uchi, T., Nakagawa, T., Miki, H., Horikawa, Y., Fujiwara, S., and Furuse, M.1997. MR angiography of intracranial aneurysms: A comparison of $0.5 \mathrm{~T}$ and $1.5 \mathrm{~T}$ Comput. Med. Imaging Graph. 21:111-116.

Locksley, H.B. 1969. Natural history of subarachnoid hemorrhage, intracranial aneurysms and arteriovenous malformations. In Intracranial Aneurysms and Subarachnoid Hemorrhage: A Cooperative Study (A.L. Sahs, G.E. Perret, and H.R. Locksley, eds.) pp. 37-108. Lippincott, Philadelphia.

Nakagawa, T. and Hashi, K. 1994. The incidence and treatment of asymptomatic, unruptured cerebral aneurysms. J. Neurosurg. 80:217-223.

Rinkel, G.J.E., Djibuti, M., Algra, A., and van Gijn, J. 1998. Prevalence and risk of ruptured of intracranial aneurysms: A systematic review. Stroke 29:251-256.

Rordorf, G., Koroshotz, W.J., Copen, W.A., Gonzalez, G., Yamada, K., Schaefer, P.W., Schwamm, L.H., Ogilvy, C.S., and Sorensen, A.G. 1999. Diffusion-weighted MR imaging: Diagnostic accuracy in patients within 6 hours of stroke symptom onset. Radiology 210:155-162.

Rosenorn, J., Eskesen, V., and Schmidt, K. 1988. Unruptured intracranial aneurysms: An assessment of the annual risk of rupture based on epidemiological and clinical data. Br. J. Neurosurg. 2:369-378.

Shellock, F.G. 1996. Pocket Guide to MR Procedures and Metallic Objects. Lippincott-Raven, Philadelphia.

Singer, M.B., Atlas, S.W., and Drayer, B.P. 1998. Subarachnoid space disease: Diagnosis with fluid-attenuated inversion-recovery MR imaging and comparison with gadolinium-enhanced spin-echo MR imaging-blinded reader study. Radiology 208:417-422.

Whisnant, J.P., Phillips, L.H., and Sundt, T.M. 1982. Aneurysmal subarachnoid hemor-rhage: Timing of surgery and mortality. Mayo Clin. Proc. 57:471-475.

Wiesmann, M., Mayer, T.E., Medele, R., and Bruckmann, H. 1999. Diagnosis of acute subarachnoid hemorrhage at 1.5 Tesla using proton-density weighted FSE and MRI sequences. Radiologe 39:860-865.

Wilcock, D., Jaspan, T., Holland, I., Cherryman, G., and Worthington, B. 1996. Comparison of magnetic resonance angiography with conventional angiography in the detection of intracranial aneurysms in patients presenting with subarachnoid haemorrhage. Clin. Radiol. 51:330-334.

Winn, H.R., Richardson, A.E., and Jane, J.A. 1977. The long-term prognosis in untreated cerebral aneurysms, I: The incidence of late hemorrhage in cerebral aneurysms: 10-year evaluation of 364 patients. Ann. Neurol. 1:358-370.

Yasui, N., Suzuki, A., Nishimura, H., Suzuki, K., and Abe, T. 1997. Long-term follow-up study of unruptured intracranial aneurysms. Neurosurgery 40:1155-1160.

Contributed by P. Ellen Grant, R. Gilberto Gonzalez, and Pamela W. Schaefer Massachusetts General Hospital Boston, Massachusetts
Rule Out (R/O) Intracranial Aneurysm

A1.2.12

Supplement 6
Current Protocols in Magnetic Resonance Imaging 